\title{
Association between dietary fat intake and MRI-determined visceral, subcutaneous, or hepatic fat in men and women from the general population
}

\author{
Christa Meisinger ${ }^{1,2}$, Susanne Rospleszcz ${ }^{3}$, Elke Wintermeyer ${ }^{4}$, Roberto Lorbeer ${ }^{5}$, \\ Barbara Thorand ${ }^{6,7}$, Fabian Bamberg ${ }^{8}$, Annette Peters ${ }^{9,7}$, Christopher Schlett ${ }^{10}$ and \\ Jakob Linseisen ${ }^{1,11}$ \\ ${ }^{1}$ Chair of Epidemiology, LMU Munich, Munich, Germany, \\ ${ }^{2}$ Independent Research Group Clinical Epidemiology, Helmholtz Zentrum München, Munich, Germany, \\ ${ }^{3}$ Institute of Epidemiology, Helmholtz Zentrum München, Munich, Germany, \\ ${ }^{4}$ Siegfried Weller Institute for Trauma Research, BG Trauma Center Tuebingen, Eberhard-Karls University Tuebingen, \\ Tübingen, Germany, \\ ${ }^{5}$ Department of Radiology, Ludwig Maximilians University Hospital, Munich, Germany, \\ ${ }^{6}$ Institute of Epidemiology, Helmholtz Zentrum München, German Research Center for Environmental Health, Munich, \\ Germany, \\ ${ }^{7}$ German Center for Diabetes Research (DZD), Munich, Germany, \\ ${ }^{8}$ Department of Radiology, University of Freiburg, Freiburg, Germany, \\ ${ }^{9}$ Institute of Epidemiology, Helmholtz Zentrum München, German Research Center for Environmental Healt, Munich, \\ Germany, \\ ${ }^{10}$ Department of Radiology, Diagnostic and Interventional Radiology, University of Heidelberg, Heidelberg, Germany \\ and \\ ${ }^{11}$ Independent Research Group Clinical Epidemiology, Helmholtz Zentrum München, Munich, Germany
}

\begin{abstract}
Introduction: An increase in dietary fat intake - at the expense of dietary carbohydrate intake - may play an important role in the accumulation of adipose tissue at different sites and liver fat accumulation. Thus, the present study investigated the isocaloric substitution of dietary carbohydrates with fat, and its cross-sectional association with visceral adipose tissue (VAT), subcutaneous adipose tissue (SAT), and hepatic fat content as determined by MRI.
\end{abstract}

Material and Methods: Data from 283 participants (mean age $56.1 \pm 9.0$ years) from the KORA FF4 study study who underwent whole-body MRI were included. VAT, SAT, and total body fat were quantified by a volume-interpolated VIBE-T1w-Dixon MR sequence. Hepatic fat content was determined as the proton density fat-fraction (PDFF) derived from multiecho-T1w MR sequence. Habitual dietary intake was estimated by combining the information provided by repeated 24-h food lists and a food frequency questionnaire. Cross-sectional associations were analyzed using linear regressions.

Results: Carbohydrate intake (in \% of total energy intake $(\mathrm{E} \%)$ ) correlated significantly inversely with VAT $(\mathrm{r}=-0.34)$ and hepatic fat $(\mathrm{r}=-0,30)$, while fat intake $(\mathrm{E} \%)$ correlated positively with hepatic fat content $(\mathrm{r}=0.16)$. Replacing total carbohydrates with an isocaloric amount of total fat was significantly positively associated with VAT and hepatic fat, while there was no significant association with SAT. The multivariable adjusted $\beta$-coefficient for replacing $5 \mathrm{E} \%$ carbohydrates with total fat was 0.39 (95\% CI: $0.02,0.76)$ for VAT. An increase in total fat intake by $5 \%$ of total energy was associated with an increase in liver fat content by $24 \%$. Dietary fiber intake was significantly inversely associated with VAT and hepatic lipid content.

Discussion: In middle-aged adults, substitution of carbohydrates with total fat was associated with a higher volume of VAT and an increase of hepatic fat, while whole-grain associated carbohydrates show inverse associations. If reproduced in prospective studies, such findings would strongly argue for limiting dietary fat intake.

\section{Conflict of Interest}

There is no conflict of interest 